
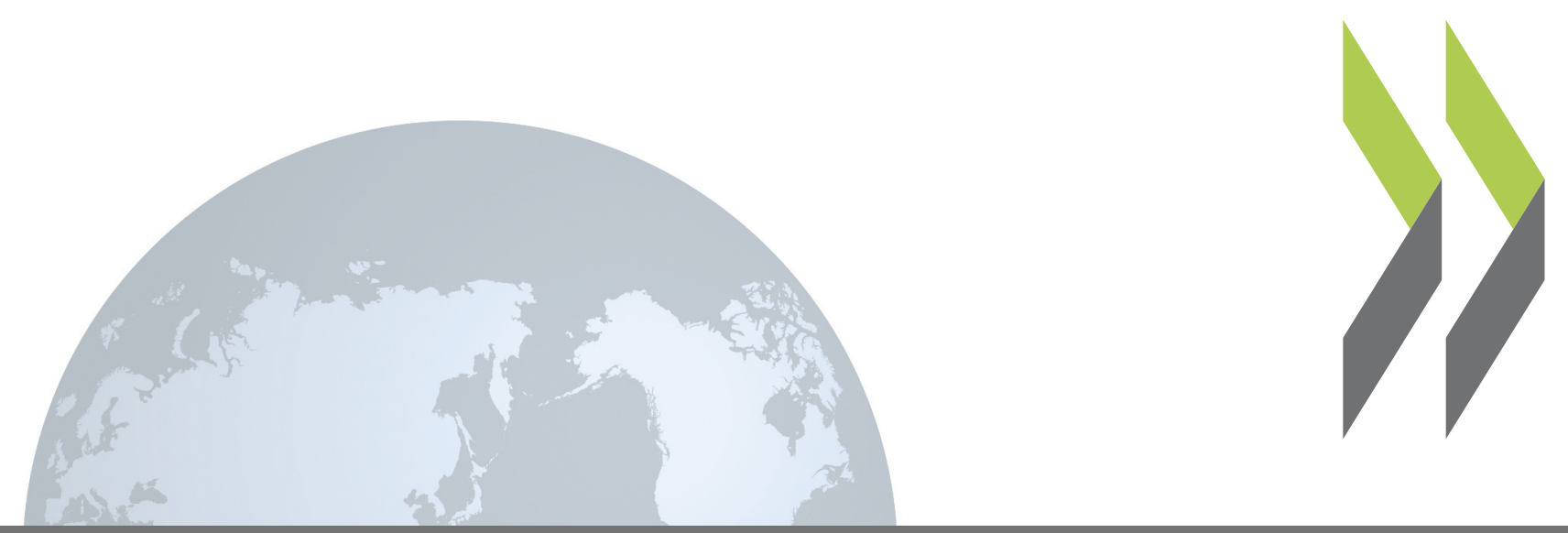

Documents SIGMA No. 1

\title{
Un corps de cadres supérieurs
} de l'administration centrale :

\section{La mise en place d'un} système de gestion pour la haute fonction publique dans les pays d'Europe centrale et orientale

\section{OCDE}


DIFFUSION GÉNÉRALE

OCDE/GD(95)45

SOUTIEN À L'AMÉLIORATION DES INSTITUTIONS PUBLIQUES ET DES SYSTÈMES DE GESTION DANS LES PAYS D'EUROPE CENTRALE ET ORIENTALE

INITIATIVE CONJOINTE DE L'OCDE/CCET ET DE LA CE/PHARE

DOCUMENTS SIGMA : $\mathbf{N}^{\circ} \mathbf{1}$

UN CORPS DE CADRES SUPÉRIEURS DE L'ADMINISTRATION CENTRALE : LA MISE EN PLACE D'UN SYSTÈME DE GESTION POUR LA HAUTE FONCTION PUBLIQUE DANS LES PAYS D'EUROPE CENTRALE ET ORIENTALE

ORGANISATION DE COOPÉRATION ET DE DÉVELOPPEMENT ÉCONOMIQUES Paris 1995

DOCUMENT DISPONIBLE SUR OLIS EN TOTALITÉ, DANS SON FORMAT D'ORIGINE 

Le Programme SIGMA -- Soutien à l'amélioration des institutions publiques et des systèmes de gestion dans les pays d'Europe centrale et orientale -- est une initiative conjointe du Centre de l'OCDE pour la coopération avec les économies en transition (CCET) et le Programme Phare de l'Union européenne. SIGMA soutient les efforts de réforme des administrations publiques déployés en Europe centrale et orientale. Il est financé à 75 pour cent par Phare et plusieurs pays Membres de l'OCDE fournissent également des ressources.

L'OCDE -- Organisation de Coopération et de Développement Economiques -- est une organisation intergouvernementale réunissant 25 démocraties à économie de marché avancée. Le CCET transmet les conseils et l'assistance de l'OCDE concernant les problèmes économiques les plus divers aux pays d'Europe centrale et orientale et de l'ancienne Union soviétique engagés dans des réformes. Le Programme Phare est une initiative de l'Union européenne qui met des compétences et des investissements à la disposition de l'Europe centrale et orientale pour y appuyer le développement d'économies de marché et de sociétés démocratiques.

Créé en 1992, SIGMA relève du Service de la gestion publique de l'OCDE (PUMA). Le PUMA fournit des informations et des analyses d'experts de la gestion publique aux décideurs des pays Membres de l'OCDE et facilite les contacts et les échanges d'expériences entre responsables de la gestion du secteur public. Par l'intermédiaire du PUMA, SIGMA offre à onze pays d'Europe centrale et orientale les précieuses connaissances techniques accumulées au cours de nombreuses années d'étude et d'action.

Les gouvernements participants et le Secrétariat de SIGMA collaborent d'une façon flexible afin de créer des programmes de travail conçus pour rendre les gouvernements mieux à même d'améliorer la gestion publique conformément à leurs priorités propres et à la mission de SIGMA. Cette initiative fait appel à un réseau international de responsables expérimentés de l'administration publique pour fournir des services de conseil et une analyse comparative des différents systèmes de gestion. Par ailleurs, SIGMA collabore étroitement avec d'autres donneurs internationaux afin de promouvoir la réforme administrative et la démocratie en Europe centrale et orientale.

Dans tous ses travaux, SIGMA s'emploie en priorité à faciliter la coopération entre les gouvernements des pays en transition. A cette fin, SIGMA accorde notamment un soutien logistique à la formation de réseaux rassemblant les praticiens de l'administration publique en Europe centrale et orientale et leurs homologues des pays Membres de l'OCDE.

Les activités de SIGMA se répartissent entre six domaines : réforme des institutions publiques, gestion de la formulation des politiques, gestion des dépenses, gestion des services publics, supervision administrative et services d'information.

\section{Copyright OCDE, 1995}

Les demandes de reproduction ou de traduction totales ou partielles doivent être adressées à : M. Le Chef du Service des Publications, OCDE, 2 rue André-Pascal, 75775 Paris Cédex 16, France.

Les idées exprimées dans la présente publication ne représentent pas les avis officiels de la Commission, des pays Membres de l'OCDE ni des pays d'Europe centrale et orientale participant au Programme. 


\section{PRÉAMBULE}

La série des Documents SIGMA est une nouvelle série de rapports spécialisés établis à titre de contribution aux travaux de SIGMA en Europe centrale et orientale et axés sur des problèmes particuliers de gestion publique tels que le contrôle des dépenses, la supervision administrative, la coordination interministérielle et la gestion des services publics. Le document intitulé Un corps de cadres supérieurs de l'administration centrale : la mise en place d'un système de gestion pour la haute fonction publique dans les pays d'Europe centrale et orientale traite d'une question d'actualité dans le domaine de la gestion des services publics.

SIGMA a établi ce document pour aider les pays à déterminer s'il serait possible de créer un certain type de corps de hauts fonctionnaires structuré selon des principes communs. Dans les pays d'Europe centrale et orientale comme dans les pays Membres de l'OCDE, les hauts fonctionnaires jouent un rôle crucial pour élever le niveau de professionnalisme de l'administration ainsi que pour réformer celle-ci dans son ensemble.

La plupart des pays d'Europe centrale et orientale n'ont pas adopté de lois relatives à la fonction publique. Toutefois, des politiques approuvées concernant le recrutement, la sélection et les transferts des hauts fonctionnaires ainsi que le déroulement de leur carrière peuvent limiter les risques de politisation de la fonction publique aux postes qui devraient être confiés à des cadres. Dans un contexte caractérisé par l'absence d'une législation de la fonction publique définissant les droits et devoirs des fonctionnaires, la création d'un corps de cadres supérieurs (CCS) peut être un facteur de stabilité.

Dans beaucoup de pays en transition, les changements de gouvernement impliquent des changements de personnel d'une ampleur trop importante pour être motivée même par des raisons politiques. Ces changements semblent avoir un caractère aléatoire, à la différence de ce que l'on observe dans les pays Membres de l'OCDE, où des changements de personnel ont bien lieu lors des changements de gouvernement, mais sous des formes généralement prévisibles et reconnaissables.

Ce document ne prétend pas définir un concept de CCS d'une portée universelle. En fait, la création d'un CCS est une tâche qui doit être entreprise dans les pays partisans de se doter d'un corps de hauts fonctionnaires de ce type compte tenu des objectifs et des conditions préalables propres au pays en question. La présente publication vise plutôt à apporter une aide pratique aux pays qui effectuent les analyses nécessaires et préparent les décisions à prendre.

Un CCS serait probablement utile dans la plupart des pays SIGMA, en particulier là où une législation relative à la fonction publique fait défaut. En pareil cas, un CCS pourrait constituer une solution pratique pour disposer plus rapidement d'une administration plus stable.

Le Professeur Jacques Ziller, Directeur de recherche à l'Institut français international d'administration publique (IIAP) a rédigé ce document pour un exposé lors de la réunion d'octobre 1994 du Groupe de liaison de SIGMA. Produit sous la forme d'un document SIGMA, ce document est maintenant à la disposition d'un public plus large.

Ce document est également disponible en anglais sous le titre Top Management Service in Central Government: Introducing a System for the Higher Civil Service in Central and Eastern European Countries.

Derry Ormond

Chef du Service de la gestion publique, OCDE 
0. Quelques remarques à l'arrière-plan $\ldots \ldots \ldots \ldots \ldots \ldots \ldots \ldots \ldots \ldots \ldots \ldots \ldots \ldots \ldots$

1. Le concept de Corps de Cadres Supérieurs $($ CCS) $\ldots \ldots \ldots \ldots \ldots \ldots \ldots \ldots \ldots \ldots \ldots$

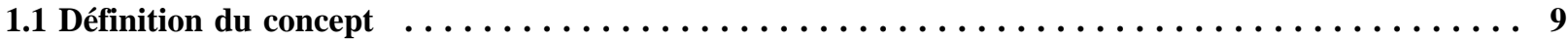

1.2 Les expériences en matière de mise en place de Corps de Cadres Supérieurs (CCS) $\ldots \ldots \ldots \ldots$

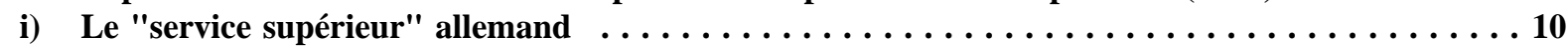

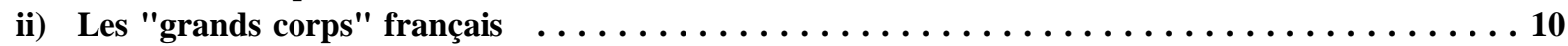

iii) Quelques expériences récentes de Senior Executive Services $\ldots \ldots \ldots \ldots \ldots \ldots \ldots \ldots \ldots$

1.3 L'apport possible d'un CCS aux fonctions essentielles de gestion

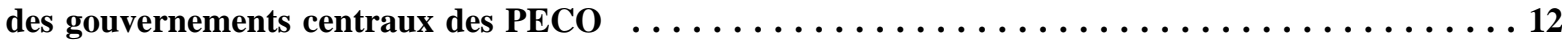

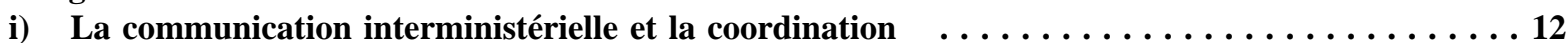

ii) Des normes, procédures et routines communes

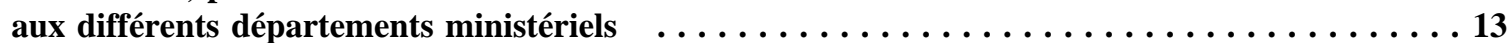

iii) Une force d'impulsion dans la fonction publique du gouvernement central $\ldots \ldots \ldots \ldots \ldots 14$

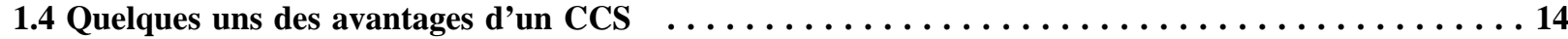

i) La possibilité de donner aux ministres des conseils à caractère neutre mais

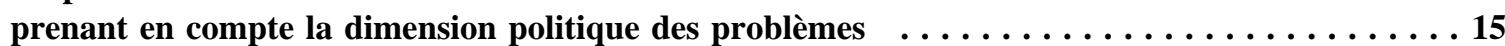

ii) Fournir la stabilité aux niveaux supérieurs de l'administration $\ldots \ldots \ldots \ldots \ldots \ldots \ldots \ldots$

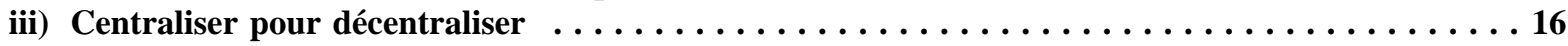

iv) Permettre un accroissement de la rémunération des fonctionnaires sans

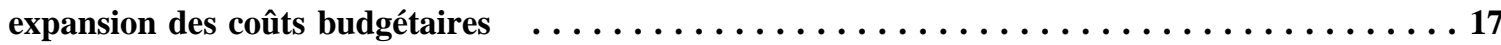

2. Les composantes nécessaires à la mise en place d'un CCS $\ldots \ldots \ldots \ldots \ldots \ldots \ldots \ldots \ldots \ldots$

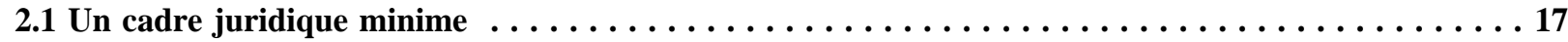

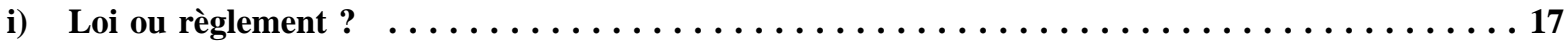

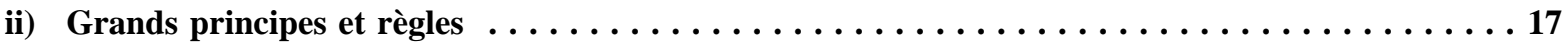

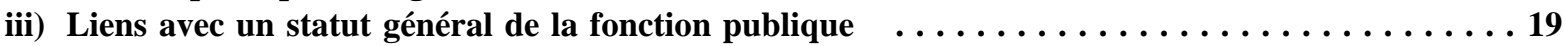

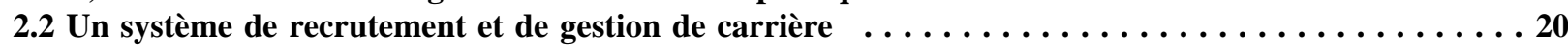

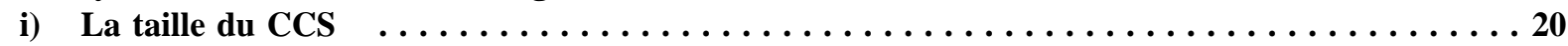

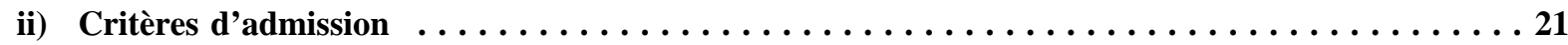

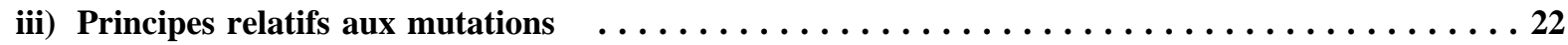

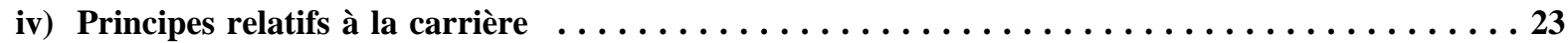

v) Des institutions pour l'admission, les mutations et la gestion des carrières $\ldots \ldots \ldots \ldots \ldots 24$

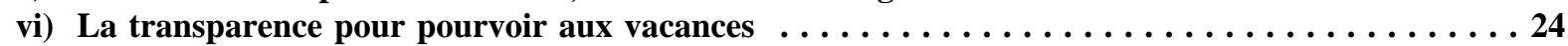

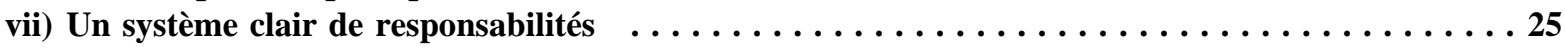

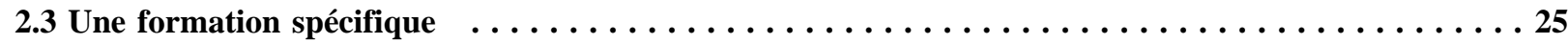

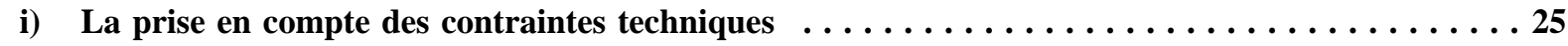

ii) L'adaptation des programmes de développement aux domaines

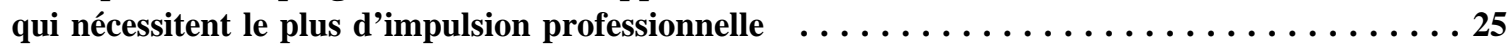

iii) Le lien entre l'évaluation et le développement des carrières $\ldots \ldots \ldots \ldots \ldots \ldots \ldots \ldots \ldots$

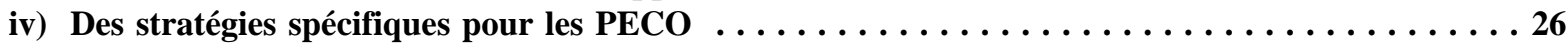

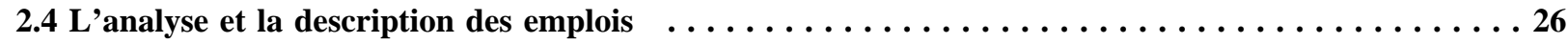

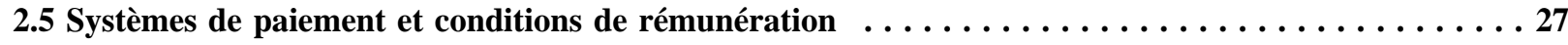

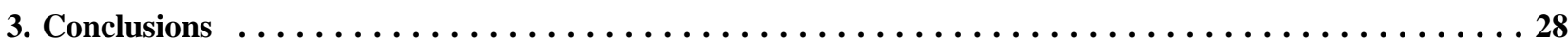

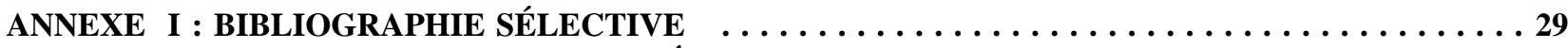




\section{INTRODUCTION}

Dans tous les pays de l'OCDE l'on considère l'existence d'une force d'impulsion professionnelle dans l'administration comme essentielle à la qualité et à l'efficacité de l'administration dans son ensemble, ainsi qu'à sa culture d'organisation.

Cette problématique a fait l'objet d'un traitement différent selon les pays. Certains, comme l'Allemagne, la France ou le Royaume-Uni, ont mis en place depuis longtemps une série de règles et traditions pour l'ensemble de la haute fonction publique, comportant des règles particulières pour la nomination aux postes supérieurs. D'autres ont créé plus récemment un corps de fonctionnaires particulier pour ces postes, comme c'est le cas du Senior Executive Service mis en place en 1978 aux États-Unis ${ }^{1}$.

Dans la plupart des pays, les personnes qui occupent les emplois supérieurs de l'administration sont nommés en général de façon centrale, par les niveaux les plus élevés du gouvernement. Ils font l'objet d'une gestion plus centralisée que l'ensemble des agents publics et fonctionnaires, parfois selon des réglementations partiellement différentes de la réglementation générale de la fonction publique. La notion de cadres supérieurs en tant qu' élite de la fonction publique, ou comme un club auquel il est désirable d'appartenir, se retrouve d'une certaine façon dans la plupart des pays occidentaux.

En faisant des efforts particuliers pour la sélection et la gestion des cadres supérieurs, les gouvernements poursuivent des objectifs de stabilisation, de professionnalisme, d'attraction pour les plus hauts niveaux de leur personnel, ainsi que de qualité et de cohérence des décisions de politiques publiques.

Une série de questions doivent être prises en compte lors de l'établissement d'un système de fonction publique :

-- doit-on distinguer entre la haute fonction publique et la masse des fonctionnaires, ou doit-on établir un système intégré ?

-- la fonction publique doit-elle être entièrement neutre à l'égard des partis politiques ou bien les libertés publiques requièrent-elles la transparence en matière d'opinions politiques des fonctionnaires ?

-- les agents publics doivent-ils être organisés en fonction du système de carrière, ou bien doit-il simplement y avoir un système de recrutement sur emplois, sans règles de promotion organisées?

-- l'administration a-t-elle principalement besoin de généralistes ou plutôt de spécialistes (de quel type) ? Quelles sont les différences entre généralistes et spécialistes en matière de formation et de carrière si tous deux coexistent ?

-- les cadres supérieurs de la fonction publique devraient-ils être gérés de manière centrale pour l'ensemble du gouvernement, ou devraient-ils être gérés sur une base sectorielle par chaque ministère ?

1 Le mot executive qui est utilisé en Amérique du Nord et dans le Pacifique pour désigner les cadres supérieurs, peut prêter à confusion. Au Royaume-Uni et en Irlande, le même mot est utilisé pour les catégories inférieures de la fonction publique : l'executive class, recrutée à un niveau d'études inférieur au baccalauréat. De plus, l'adjectif senior risque d'induire des confusions avec la notion d'ancienneté.

C'est pourquoi la version anglaise de ce document utilise l'expression top managers ou top public managers pour désigner les cadres supérieurs (de la fonction publique). 
Il existe d'importantes corrélations entre les types de réponses à ces questions, comme le montre le système de Corps de Cadres Supérieurs (CCS) étudié ici. Dans un contexte de transition, la mise en place d'institutions clairement définies et de procédures et instruments de gestion des cadres supérieurs, pourrait être une voie rapide pour arriver à la stabilisation et au professionnalisme de l'administration, de même que pour faciliter la mise en œuvre des réformes.

Le présent document met en lumière les questions à examiner pour la mise en place d'un système de gestion des cadres supérieurs dans l'administration centrale des pays d'Europe centrale et orientale (PECO) et indique les pistes de possibles solutions pratiques, qui pourraient être mises en œuvre par les gouvernements intéressés.

\section{Quelques remarques à l'arrière-plan}

0.1. Les PECO ont hérité d'une structure administrative de type soviétique, avec des ministères sectoriels non coordonnés, qui gèrent directement leurs activités jusqu'à l'échelon le plus bas. Les décisions politiques et stratégiques, y compris en matière budgétaire, étaient approuvées par le Parti et non préparées ou coordonnées par le Conseil des ministres, ou décidées par le Parlement. Le centre du gouvernement, à savoir le Conseil des ministres, le Premier ministre et le ministres des Finances, n'avaient pas l'autorité en matière de décisions politiques et de finances qu'ils ont d'habitude dans les gouvernements d'Europe de l'Ouest. L'adoption d'un système multi-parti et de systèmes parlementaires de type occidental (certains pays donnant un rôle important au Président de la République, comme c'est aussi le cas en France, en Finlande ou au Portugal), n'a pas supprimé pour autant cet les effets de cet héritage.

0.2. Étant donné que la réforme de l'administration centrale et l'adoption d'une structure démocratique sont des processus à long terme, beaucoup de ces traits continuent à prévaloir, à l'exception du rôle Parti comme coordinateur et décideur ultime.

Dans ce contexte, il peut être utile de mentionner les points suivants, qui sont applicables d'une certaine manière à tous les PECO, même si existent d'importantes différences de pays à pays :

i) La structure sectorielle n'est pas encore brisée, et les ministères sectoriels avec leur administration, traditionnellement importante, restent très puissants par comparaison avec le centre du gouvernement. Dans le régime précédent, les ministères sectoriels s'adressaient au Parti sans consulter les autres départements ministériels afin de faire approuver les décisions qu'ils avaient préparées. Il y a un danger que cette procédure manquant de cohésion ne se perpétue avec la nouvelle structure gouvernementale.

Chaque ministre a le pouvoir d'organiser son ministère à sa convenance. Contrairement au cas des pays occidentaux, ce pouvoir ne concerne pas seulement les fonctions d'exécution et la structure interne du ministère, mais aussi les parties impliquées dans le processus de décision du gouvernement central.

ii) En général les liens entre différents ministères et les structures et procédures de coopération et de négociation interministérielles pour les décisions gouvernementale ne sont pas encore pleinement développées.

Le processus législatif n'est pas encore d'une qualité suffisante, même si le nombre de projets de loi est très important. Les projets sont élaborés dans les ministères sectoriels sans procédures appropriées pour la consultation d'autres ministères, en particulier du ministère des Finances, ou d'autres organismes compétents externes à l'administration. 
Le ministère des Finances n'a pas encore un rôle approprié dans la préparation du budget de l'ensemble du gouvernement. La procédure budgétaire en tant que fonction commune, comme dans les pays occidentaux, n'existe pas encore.

iii) L'administration est encore très influencée par la politique partisane ; les personnes au sommet de l'administration vont et viennent au gré des ministres, et changent très souvent.

La séparation entre fonctions administratives et politiques n'est pas poussée au bout de sa logique, ce qui ne permet pas de savoir quels sont les postes dont le sort est lié à celui du gouvernement et lesquels ont un statut permanent.

Les fonctionnaires, y compris les cadres supérieurs, sont employés par les ministères sectoriels, non par le gouvernement, ce qui renforce la sectorisation déjà indiquée. Il n'y a pas de gestion en commun du personnel et il n'y a pas de mécanismes pour la mobilité entre ministères.

Même lorsqu'une loi portant statut de la fonction publique a été adoptée, les schémas formels de carrière ne se réfèrent qu'aux diplômes et à l'ancienneté, et n'encouragent pas la promotion des personnes les plus compétentes et les plus dynamiques.

iv) Les collectivités locales ont un champ d'action et des ressources très limités. La plupart des activités administratives locales sont encore organisées au sein des structures des ministères sectoriels de l'Etat, même si la décentralisation et la réforme territoriale sont à l'ordre du jour de la réforme dans la plupart des pays.

0.3. Le développement de fonctions de gestions transversales, communes à tous les ministères (budget de l'Etat, processus de décision politique) ou gérées selon des normes communes (personnel, travail législatif), est à l'ordre du jour de la réforme administrative dans la plupart des pays. Un exemple est celui des réformes de la fonction publique, qui sont préparées ou mises en œuvre dans les PECO. L'objectif de telles réformes est de mettre fin aux ingérences de politique partisane dans l'administration et d'assurer la mise en place d'une fonction publique professionnelle et permanente de qualité égale dans tous les ministères. Même si les progrès peuvent être lents, la conséquence en sera un transfert de pouvoirs des ministères sectoriels vers le centre de gouvernement.

0.4. La problématique à étudier dans ce contexte d'héritage et de développement actuel des réformes est de savoir si la mise en place d'un Corps de Cadres Supérieurs (CCS) pourrait faciliter le développement des nécessaires fonctions de gestion transversales du gouvernement, augmenter la qualité et l'efficacité du gouvernement central et de son processus de décision, et permettre une amélioration du processus global de gestion des ressources humaines de l'administration, même si la législation applicable à la fonction publique n'a pas encore été adoptée.

\section{Le concept de Corps de Cadres Supérieurs (CCS)}

\subsection{Définition du concept}

Afin de clarifier le concept pour les décideurs politiques et les fonctionnaires des pays d'Europe centrale et orientale, la définition suivante d'un CCS est proposée :

Un Corps de Cadres Supérieurs (CCS) est un système, structuré et reconnu, de gestion du personnel pour les postes non politiques les plus élevés de l'administration (postes de cadres supérieurs de l'administration). Il s'agit d'une fonction publique de carrière, apte à fournir des cadres 
formés et expérimentés pour être nommés à ces postes. Le corps est géré de manière centrale par des institutions et procédures appropriées, afin de garantir la stabilité et le professionnalisme du groupe central des cadres supérieurs, mais en permettant la flexibilité nécessaire pour répondre aux changements dans la composition du gouvernement.

\subsection{Les expériences en matière de mise en place de Corps de Cadres Supérieurs (CCS)}

Plusieurs expériences dans les pays de l'OCDE peuvent être directement ou plus souvent indirectement rattachées au concept de CCS.

i)

Le "service supérieur" allemand (Höherer Dienst)

La fonction publique allemande est toujours organisée selon les principes établis au début du XVIIIème siècle pour garantir le professionnalisme des fonctionnaires et leur neutralité dans le service au public. Le "service supérieur" regroupe tous les fonctionnaires recrutés au terme d'études universitaires (5 ans et demi d'études théoriques et pratiques et deux difficiles examens d'Etat). Les statuts de la fonction publique (loi fédérale et lois des Länder ainsi que plusieurs décrets) fixent les conditions nécessaires à la promotion des membres du service supérieur aux postes les plus élevés des ministères fédéraux et de Länder). Toutefois des règles spécifiques permettent au gouvernement d'écarter (en les mettant à la "retraite provisoire") les titulaires des postes des deux niveaux les plus élevés dans les ministères ${ }^{2}$. Les Secrétaires d'Etat (en Allemagne cette appellation est donnée aux fonctionnaires de carrières qui occupent le poste au sommet de la hiérarchie d'un ministère, dans d'autres pays, le terme utilisé est souvent celui de Secrétaire général) et directeurs (Abteilungsleiter) qui ne conviennent pas au ministre peuvent ainsi être remplacés par d'autres membres du "service supérieur".

Afin de permettre une plus grande flexibilité des carrières des membres du service supérieur, le Land de Bade-Württenberg a mis en place en 1986 une Académie des cadres du service public afin de créer une élite de cadres supérieurs de l'administration.

\section{ii) Les "grands corps" français}

Le concept de corps, regroupant les fonctionnaires appartenant à une même carrière, et qui n'est connu dans la plupart des pays qu'en ce qui concerne le corps diplomatique, est le principe d'organisation de la fonction publique en France depuis le début du XIXème siècle (et plus récemment en Espagne). Il faut mentionner en particulier le Conseil d'État (à la fois conseiller juridique du gouvernement et cour administrative suprême), la Cour des Comptes et l'Inspection des finances. Ces corps ont trois à quatre fois plus de membres qu'il ne serait nécessaire pour occuper les fonctions qui leur sont propres, afin de constituer un réservoir de cadres pour les postes supérieurs de l'administration. Les membres de ces corps peuvent être en poste selon les cas dans leur institution d'origine ou dans les ministères et établissement publics de l'Etat où ils peuvent exercer les fonctions de directeurs ou sous-directeurs. La même chose vaut pour les corps d'ingénieurs recrutés par l'École polytechnique et les écoles d'application telles que les Ponts et chaussées, les Mines etc.

Le projet lié à l'origine à la mise en place de l'École nationale d'administration (ENA) en 1945 a des caractéristiques qui le rapprochent des expériences plus récentes de CCS. La réforme avait pour but la démocratisation et le renouvellement des élites de l'administration ainsi que son décloisonnement. L'idée principale de la réforme était le recrutement et la formation en commun des grands corps déjà existants

\footnotetext{
${ }^{2}$ L'appellation de "fonctionnaires politiques" (politische Beamte) donnée aux titulaires de ces postes depuis plus d'un siècle (le système est décrit par Max Weber dans ses ouvrages relatifs à l'administration publique) ne doit pas être interprétée à tort : il s'agit bien de postes administratifs et non politiques.
} 
(autres que ceux d'ingénieurs) ainsi que la création d'un nouveau corps d'administrateurs civils destinés à occuper les principales fonctions de direction des ministères et établissements publics. Contrairement aux corps existants, qui avaient été établis sur une base sectorielle, le nouveau corps était interministériel, afin de permettre une plus grande mobilité des généralistes. La formation initiale donnée à l'ENA aurait dû être une partie intégrante d'un système de formation incluant la formation permanente qui devait être dispensée par le Centre des hautes études administratives. En combinaison avec le recrutement interne de l'ENA (qui est organisé par un concours séparé réservé aux fonctionnaires déjà expérimentés) ceci aurait mise en place un véritable système de développement des cadres.

Le mécanisme permettant la stabilité du groupe central des cadres supérieurs de l'administration et la mobilité aux postes les plus élevés, repose sur la séparation entre le grade (position de carrière dans le corps) et l'emploi (poste temporaire dans un ministère ou établissement public de l'Etat).

\section{iii) Quelques expériences récentes de Senior Executive Services}

L'exemple le mieux connu d'un CCS est sans doute le Senior Executive Service (SES) mis en place dans l'administration fédérale des États-Unis par la loi de réforme de la fonction publique de 1978.

Le SES fédéral américain comprend un groupe d'à peu près 8000 personnes occupant les postes des trois niveaux les plus élevés de l'administration. 85 pour cent des membres du SES doivent être des fonctionnaires de carrière, seuls 10 pour cent peuvent être nommés à titre temporaire, les 5 pour cent restant correspondant à des nominations d"'urgence" ou de très courte durée. De nombreux postes ne peuvent être confiés qu'à des fonctionnaires de carrière (40 pour cent du total des postes concernés). Il n'y a pas de lien automatique entre le grade d'un membre de la fonction publique et son emploi. Les nominations aux emplois sont prononcées par le Président des États-Unis ou par les chefs d'agences, et les mutations ne peuvent être prononcées qu'avec un préavis de deux semaines, à l'exception d'une période de 120 jours après l'installation d'une nouvelle "administration" présidentielle ou la nomination d'un nouveau chef d'agence. La loi de réforme de 1978 a mis en place un système d'évaluation de l'efficacité et de paiement au mérite. La carrière des membres du SES est le résultat des postes successifs qu'ils occupent ainsi que des programmes de développement des cadres qu'ils suivent. L'Office of Personnel Management a été chargé d'établir des réglementations relatives aux qualifications nécessaires pour accéder au SES, de mettre en place des commissions d'examen des qualifications des personnes nommées dans les agences pour leur entrée dans le SES, ainsi que de mettre en place, ou de faire mettre en place par les agences, des programmes permettant le développement systématique des candidats à l'entrée dans le SES. Il y a un lien direct entre les programmes de développement des cadres et le choix des titulaires de postes vacants.

Selon la plupart des commentaires, le SES fédéral américain n'a pas tenu toutes ses promesses du fait des contradictions internes au système établi par la loi de réforme de la fonction publique et du fait de la politisation des nominations et récompenses par l'"administration" présidentielle.

D'autres gouvernements, en particulier ceux des États fédérés américains, d'Australie et de Nouvelle-Zélande, on mis en place des SES au début des années quatre-vingt, s'inspirant plus ou moins de l'expérience fédérale américaine. Au même moment, le Canada a mis en place la Catégorie exécutive. Selon des idées approchantes, l'Italie a établie un "corps directif" (dirigenza) dès 1972. Dans les années soixante-dix et quatre-vingt, le Royaume-Uni, tout en gardant le système traditionnel de nomination aux postes supérieurs de l'administration sur la base d'un certificat délivré par la Civil Service Commission, a mis en place des carrières spécifiques liées à une formation particulière pour les jeunes hauts fonctionnaires les plus prometteurs, avec le "fast stream" et l'"european fast stream". La Belgique, et plus récemment 
les Pays-Bas, ont lancé des expériences de développement des cadres ; en Espagne les gouvernements de certaines communautés autonomes, telles que le Pays Basque, se sont engagé dans des voies semblables ${ }^{3}$.

\subsection{L'apport possible d'un CCS aux fonctions essentielles de gestion des gouvernements centraux des PECO}

L'actuelle sectorialisation de l'administration des PECO rend nécessaire la mise en place de structures et procédures destinées à renforcer la coordination de la prise de décision et de la mise en œuvre des politiques. La transition vers l'économie de marché et vers des institutions démocratiques comprenant une administration forte rend d'autant plus nécessaire une force d'impulsion dans la fonction publique, afin de permettre l'application des changements. Le présent paragraphe étudie ces fonctions et l'apport que peut réaliser un CCS à leur exercice.

\section{i) La communication interministérielle et la coordination}

La plupart des PECO ont adopté des institutions parlementaires, ou un mélange de système parlementaire et de Président de la République fort. De même qu'en Europe de l'Ouest, le système parlementaire nécessite une coordination renforcée au niveau de l'administration, du fait qu'il n'y a pas de ligne hiérarchique directe entre le chef de gouvernement et les gestionnaires des départements ministériels, et parce que les coalitions multi-parti ne renforcent pas d'habitude l'action collective du gouvernement. Quel que soit le degré de solidarité entre les membres du gouvernement, la communication et la coordination interministérielle -- fondamentales en elles-mêmes pour la prise de décision et la mise en œuvre des politiques publiques -- ne peuvent être exercées par le seul conseil des ministres ou même par des réunions interministérielles au niveau des ministres. La communication et la coordination doivent être complétées au niveau des fonctionnaires.

La coordination implique différentes actions, procédures et structures. Dans un gouvernement moderne, il n'y a guère de domaine de politique où l'action isolée d'un département ministériel ou d'un établissement d'Etat peut se prolonger longtemps.

C'est le cas en particulier des procédures budgétaire et législative. L'élaboration d'un budget est une procédure longue (huit à neuf mois dans les pays d'Europe de l'Ouest) de communication entre le ministère des Finances et les ministères dépensiers, qui conduit à une série d'arbitrages entre les ministères dépensiers. Le ministre des Finances ne peut arbitrer que s'il a une position forte du point de vue technique et politique. Même dans ce cas, il doit y avoir au bout du compte des arbitrages entre les ministères dépensiers et le ministère des Finances, qui ne peuvent être prononcés que par le Premier ministre ou le Conseil des ministres. Pour que le système soit efficace, seul un nombre limité d'arbitrages budgétaires doit être prononcé par les ministres eux-mêmes : dans la plupart des pays d'Europe de l'Ouest, près de 95 pour cent de ces décisions sont le résultat d'un processus itératif entre départements ministériels. La plupart de ces décisions sont prises par les fonctionnaires chargés de la préparation du budget et par les cadres supérieurs des ministères, et il n'y a qu'un nombre limité de cas dans lesquels les ministres interviennent eux-mêmes.

En ce qui concerne le processus législatif, la coordination est également nécessaire à tous les départements ministériels, pour plusieurs raisons : le Parlement n'a qu'une capacité limitée d'examen sérieux et approfondi des projets gouvernementaux, ce qui signifie que des arbitrages doivent être faits pour la mise au point de l'ordre du jour de l'action gouvernementale et législative. De plus, les frontières entre les compétences des départements ministériels ne doivent pas et ne peuvent pas être étanches, ce qui signifie qu'une bonne communication entre les ministères au stade de l'élaboration des projets est nécessaire afin

\footnotetext{
${ }^{3}$ Voir l'Annexe I pour des références bibliographiques relatives aux systèmes de CCS.
} 
d'éviter des chevauchements ou des contradictions dans les objectifs et moyens de différentes parties de la législation. Ceci est encore plus important dans les PECO, où les compétences des départements ministériels doivent être redéfinies en fonction des différentes phases de la transition vers l'économie de marché. Dans le cas contraire, il existe un sérieux danger de voir adopter de nouvelles législations sans que l'on n'ait une vue suffisamment claire des contradictions avec la législation et la réglementation existantes, ce qui conduit à abandonner d'importants aspects de la décision politique au contrôle constitutionnel.

La plupart des conflits interministériels pourraient être résolus à un stade précoce par des consultations appropriées entre les départements concernés, ce qui permettrait une clarification des positions et des intérêts, et si nécessaire, une négociation conduisant à un accord entre fonctionnaires. L'existence de procédures d'arbitrage formelles et informelles au niveau des cadres supérieurs évite que la plupart des conflits techniques ne soient transférés au Conseil des Ministres. Cela permet un plus grand impact des directives et instructions gouvernementales puisque leur mise en œuvre dépend des fonctionnaires directement impliqués. Ce type de coordination interministérielle n'es possible que si les fonctionnaires concernés partagent une même idée de la pratique des procédures budgétaires, législatives et administratives et s'ils sont capables de concevoir l'administration comme un tout plutôt que de ne s'intéresser qu'à leur seul département. Il en va de même pour la mise en œuvre des politiques, dans la mesure où ce processus nécessite une répartition claire et une acceptation des responsabilités d'exécution, de même que des adaptations d'ordre réglementaire ainsi que dans l'allocation des ressources.

Le système administratif et la culture de la fonction publique actuels des PECO a plutôt l'effet inverse : les structures comme la culture de l'administration ont tendance à renforcer la fragmentation, car elles sont orientées vers la communication verticale et les décisions hiérarchiques sans délégation suffisante, ce qui a pour conséquence un durcissement des conflits interministériels qui, de ce fait, remontent au Conseil des ministres ou au Premier ministre. Un CCS pourrait être d'un grand secours pour changer les attitudes grâce à une formation commune, à la mobilité interministérielle et finalement à l'esprit de corps des fonctionnaires directement impliqués dans le processus de décision ministériel et interministériel.

ii)

\section{Des normes, procédures et routines communes aux différents départements ministériels}

Dans plusieurs pays de l'OCDE (en particulier au Royaume-Uni, en Australie et en Nouvelle-Zélande) l'on trouve une tendance à la fragmentation de l'administration centrale en unités plus petites et plus faciles à gérer. Ces unités reçoivent ou recevront une plus grande autonomie non seulement dans la gestion des politiques dont elles sont responsables, mais également en ce qui concerne la fixation de leurs normes, procédures et routines, puisque leur orientation vers la clientèle devrait les conduire à adapter leur réglementations à leurs besoins propres. Cette tendance ne sera pas discutée ici. Mais il est à souligner que les PECO doivent d'abord élaborer et mettre en œuvre avec continuité des normes, procédures et routines communes aux différents départements ministériels avant même que de s'interroger sur les possibles avantages de la fragmentation telle qu'elle est mise en place dans d'autres pays. Ces normes, procédures et routines communes sont nécessaires dans trois domaines essentiels au moins : la procédure budgétaire, la gestion des ressources humaines et les contrôles de légalité.

Dans une administration moderne, l'élaboration du budget du gouvernement est un long processus de négociation, dont il a déjà été fait mention. Mais il faut souligner que ce processus suit des procédures techniques très standardisées, qui doivent être partagées par toutes les parties de l'administration afin de faciliter des propositions réalistes et la confiance mutuelle lors des étapes de négociation et d'arbitrage de ce processus. Il ne s'agit pas d'une matière réservée aux seuls spécialistes. Tous les décideurs du gouvernement et de l'administration doivent avoir une interprétation claire et commune de ces normes et procédures, afin d'être capables de négocier avec talent lorsque c'est nécessaire, mais aussi, plus généralement, de garder à l'esprit les contraintes budgétaires lors de la préparation ou de l'adoption de projets politiques. 
Ici encore, la mise en place d'un CCS pourrait s'avérer un moyen rapide d'installer et de diffuser une culture et des interprétations communes à l'ensemble de l'administration.

\section{iii) Une force d'impulsion dans la fonction publique du gouvernement central}

Les PECO connaissent de très rapides changements économiques et sociaux, bien plus importants que ceux des pays de l'OCDE. Après une courte période où l'on a cru à des solutions radicales en termes de marchés, où l'administration publique n'était perçue que comme une gêne, il est à présent clairement admis que l'administration a un rôle très important à jouer à la fois pour guider le mouvement de transition et pour s'adapter à sa nouvelle fonction de gardien des règles de l'économie de marché et de l'Etat de droit. Cela implique un besoin de force d'impulsion dans l'administration, de manière à garder une ligne cohérente en matière de décision politique. Cette force d'impulsion ne peut pas être donnée par le seul sommet politique, même dans des périodes de conjoncture politique relativement stables. Comme le montrent quelques exemples historiques, tels que la Prusse après les guerres napoléoniennes ou plus récemment la France après la deuxième guerre mondiale, une fonction publique forte peut jouer un rôle important dans la restructuration des institutions et de l'économie d'un pays.

Le faible degré d'efficacité de la fonction publique est un obstacle à des politiques dotées d'un impact réel, et est encore plus un obstacle à la réévaluation du rôle de l'administration dans le processus de transition. Il faut pour ce faire un effort de réforme important, qui ne peut être couronné de succès que s'il y a une volonté de réforme claire et constante au sommet de la fonction publique. Cette clarté et continuité ne peut pas être non plus le fait du seul sommet politique. Les gouvernements de coalition, souvent accompagnés de l'instabilité gouvernementale, peuvent difficilement assurer le dynamisme continu dont a besoin le processus de changement. Mais une élite administrative solide, soutenant les efforts politiques ou les remplaçant en cas de déficience, peut permettre d'obtenir d'importants résultats, comme le montre par exemple la France de l'après-guerre, où la ré-industrialisation et l'adaptation à une économie plus ouverte se sont produites sous l'impulsion d'un système administratif dirigé avec constance par la haute fonction publique, même dans les périodes de gouvernements très faibles et éphémères.

La mise en place d'un CCS pourrait être une manière d'aboutir à une telle force d'impulsion, par la création d'un esprit de corps conforté par des valeurs de service public et des expériences communes. Si le système est bien conçu et pourvu de moyens suffisants, il peut devenir un puissant instrument pour restaurer ou promouvoir la motivation de la fonction publique, en donnant des perspectives de carrière claires aux meilleurs éléments de la fonction publique existante et en attirant des personnes extérieures ou ré-attirant d'anciens membres de la fonction publique. Ce peut être un instrument de création, de perpétuation et de solidification d'une culture administrative.

\subsection{Quelques uns des avantages d'un CCS}

Il existe différentes solutions aux problèmes actuels et futurs des administrations des PECO, et la mise en place d'un CCS ne peut être qu'une partie du processus de de réforme d'ensemble. Néanmoins, l'un des avantages liés à l'installation rapide d'un CCS est que les objectifs et moyens d'une telle réforme sont aisés à identifier, qu'il s'agit d'une partie mieux ciblée et pourtant plus large que d'autre éléments de réforme, et surtout que cela est susceptible de développer une dynamique favorable à d'autres réformes de l'administration. D'autres avantages doivent également être soulignés du fait de leur caractéristique d'effet à moyen ou long terme, qui poussent à l'adoption d'un CCS comme modalité permanente de l'administration. 
La possibilité de donner aux ministres des conseils à caractère neutre mais prenant en compte la dimension politique des problèmes

Un Corps de Cadres Supérieurs (CCS) bien conçu devrait permettre le développement d'une élite administrative de généralistes conscients des différents aspects du travail gouvernemental, capables de gérer des départements ministériels ou établissements publics ainsi que de donner au ministre des conseils prenant en compte la dimension politique des problèmes. Le CCS peut jouer un rôle que ni les conseillers plutôt inexpérimentés des ministres, ni les consultants privés — qu'ils soient nationaux ou étrangers — ne sont capables d'exercer car il leur manque une compréhension suffisante de l'appareil administratif existant et de celui qu'il conviendrait de développer.

De plus, un CCS pourrait permettre de combler le fossé qui sépare la prise de décision de la mise en œuvre des politiques, qui risque de conduire à l'élaboration de programmes politiques très irréalistes. Le CCS serait organisé de manière à développer la mobilité entre les départements ministériels centraux d'un côté et les établissements publics et services déconcentrés de l'Etat de l'autre côté. Un système adapté de mobilité entre ces différentes parties de l'administration faciliterait un apport de nouvelles idées fondées sur une bonne compréhension de l'environnement des politiques.

\section{ii) Fournir la stabilité aux niveaux supérieurs de l'administration}

Afin de permettre les changements au niveau politique, qui sont le corollaire naturel de la démocratie parlementaire, sans pour autant interrompre le fonctionnement de l'administration, il est nécessaire de trouver de la stabilité quelque part dans les niveaux supérieurs du système politico-administratif. Un CCS, fondé sur la séparation entre le grade et l'emploi, fournirait un noyau central de hauts fonctionnaires qui resteraient dans la sphère administrative quels que soient les changements de gouvernement en place.

Un système convenablement structuré pourrait essayer de concilier deux objectifs apparemment différents :

-- la réduction de l'ingérence de la politique partisane dans le recrutement et la gestion des carrières de la fonction publique, ainsi que

-- une flexibilité suffisante pour que les ministres puissent établir des relations de travail de bonne qualité et marquées par la confiance, avec le sommet administratif de leurs départements, afin de permettre l'impulsion politique nécessaire au bon fonctionnement de l'administration.

Les expériences occidentales traditionnelles indiquent ce qui devrait être évité et ce qui peut être fait en la matière.

Le système britannique de neutralité totale de la fonction publique a été critiqué récemment du fait qu'il permet aux secrétaires généraux et à leurs collaborateurs d'influencer plus fortement la politique de leur ministre que ne le fait le programme du parti au pouvoir. D'un autre côté, les restes du système des dépouilles dans l'administration présidentielle américaine ont été critiqués du fait qu'ils conduisent à des interruptions trop fortes de l'activité gouvernementale lors de chaque changement de président, et du fait qu'elle favorise l'arrivée d'un trop grand nombre de cadres supérieurs inexpérimentés dans les emplois supérieurs de l'administration. 
Le système allemand des "fonctionnaires politiques" est une solution partielle à ces contradictions, du fait qu'il permet au gouvernement de nommer des candidats extérieurs aux emplois supérieurs vacants, à condition qu'ils possèdent la qualification à devenir fonctionnaires du service supérieur. Néanmoins ce système est rigide, car le seul moyen de provoquer une vacance d'emploi est de mettre d'office son titulaire à la "retraite provisoire". Le système français a certaines caractéristiques semblables à un CCS, même si la haute fonction publique est fragmentée en une douzaine de corps. Les nominations aux emplois supérieurs de l'administration ("à la discrétion du gouvernement") peuvent être prononcées en dehors de la fonction publique. Mais ceci n'arrive que très rarement, du fait que les corps fournissent une réserve suffisamment importante de cadres expérimentés et bien formés pour que le gouvernement en place puisse y trouver des candidats appropriés pour pourvoir les emplois supérieurs. Si les ministres ne parviennent pas à établir des relations de travail confiantes avec les fonctionnaires supérieurs de leur département, ils peuvent mettre fin à leurs fonctions. Mais ils restent à l'intérieur de la fonction publique, soit avec un nouvel emploi dans un autre département ministériel ou un établissement public dont le ministre de tutelle s'entend mieux avec eux, soit dans des postes plus indépendants au sein d'organes de contrôle interne, des autorités indépendantes ou tribunaux administratifs pour lesquels une relation de travail étroite avec le ministre n'est pas nécessaire.

Une solution supplémentaire pour permettre de réconcilier la stabilité administrative et la sensibilité politique est le système des cabinets ministériels tels qu'il existe en Belgique, en France et en Italie (comme à la Commission européenne) de même que dans certains PECO. Ils consistent en un groupe de conseillers particuliers du ministre, qui sont chargés de traduire les décisions politiques du ministre en directives et instructions pour l'action administrative, ainsi que du suivi du travail des unités administratives. La nomination des membres du cabinet est habituellement laissée à la discrétion du ministre. Toutefois, en Belgique, les deux tiers des membres du cabinet doivent être choisis dans la fonction publique, afin d'en assurer la compétence technique. En France il n'y a pas de réglementation de ce type, mais en pratique plus de 90 pour cent des membres des cabinets proviennent de la fonction publique, en particulier des grands corps, et retournent dans leur administration d'origine lorsque le ministre quitte le gouvernement ou les renvoie.

Un CCS pourrait aussi devenir un réservoir de fonctionnaires techniquement compétents pour servir dans les cabinets ministériels, a condition qu'il y ait un système clair de règles assurant la transparence dans la composition et le rôle des cabinets : quel est le nombre de leurs membres ? sont-ils payés sur le budget du ministère auquel appartient le cabinet ? quelles sont les règles relatives à la délégation de pouvoir du ministre aux membres du cabinet ? etc.

C'est pourquoi un CCS est compatible à court terme avec des nominations politiques discrétionnaires aux emplois supérieurs des ministères, tout en mettant en place une structure qui à long terme garantira la compétence et l'expertise des personnes qui peuvent être nommées à ces emplois en fonction de choix discrétionnaires des ministres.

\section{iii) Centraliser pour décentraliser}

Afin de fonctionner correctement, un système de CCS devrait comprendre une institution centrale d'un type ou d'un autre, chargée de la gestion du corps. Toutefois, une telle institution centrale devrait être comprise comme un outil permettant la décentralisation réelle, car celle-ci ne peut réellement fonctionner que s'il y a un degré suffisant de culture administrative commune et une bonne communication entre unités décentralisées. Loin de conduire à plus de centralisation, une gestion centrale de la haute fonction publique deviendrait l'un des principaux instruments d'une décentralisation efficace. 
L'un des problèmes communs à de nombreux PECO est celui de la fuite des cerveaux due à la très forte différence entre les salaires des cadres du secteur privé et ceux des hauts fonctionnaires. Ceci peut aussi être un facteur dissuasif pour les meilleurs étudiants qui pourraient devenir fonctionnaires. L'existence d'un groupe de cadres supérieurs experts capables d'exercer différentes fonctions dans différents ministères pourrait permettre une réduction des effectifs globaux de hauts fonctionnaires. Les gains de productivité induits par le système pourraient conduire à la suppression de nombreux emplois supérieurs, ce qui permettrait une redistribution de la masse salariale affectée à ces emplois entre les membres du CCS. Ceci pourrait ensuite générer des améliorations de la structure salariale, ce qui rendrait la haute fonction publique plus attrayante pour les jeunes.

\section{Les composantes nécessaires à la mise en place d'un CCS}

Comme le montre la brève description des expériences concrètes de CCS, de tels systèmes sont faits d'une série de composantes qui peuvent différer de pays à pays. Le présent paragraphe essaie de définir ces composantes essentielles à la mise en place d'un CCS et indique les différentes options existant pour chacune de ces composantes.

Il faut souligner que le système doit être robuste et plutôt simple, en particulier lors des premières années suivant sa mise en place. Un système très élaboré serait à la fois fragile et trop peu transparent pour pouvoir prendre racine et permettre des développements ultérieurs.

\subsection{Un cadre juridique minime}

La mise ne œuvre d'un système de CCS nécessite une définition claire des règles ainsi qu'un horizon temporel suffisamment clair pour que le nouveau système soit attrayant et puisse être évalué après une pratique suffisante.

Dans le cas du SES fédéral américain, la loi de réforme de la fonction publique, qui ne partait d'ailleurs pas de zéro mais avait été précédée de longues discussions et études, prévoyait une évaluation au bout de cinq ans avant que le SES ne voie son existence définitivement consacrée. Dans les PECO il faut d'abord un consensus et une acceptation de la part des ministères et institutions impliquées dans une telle réforme, la législation destinée à conforter le CCS pouvant venir par la suite.

i)

\section{Loi ou règlement ?}

La question de savoir si ce cadre juridique devrait prendre la forme d'une loi ou simplement d'un règlement reste ouverte. La réponse dépend des règles constitutionnelles relatives à la compétence en matière de réglementation de la fonction publique ainsi que le la stabilité et de la capacité à générer la confiance de ces deux types d'instruments juridiques. Elle dépend aussi fortement de facteurs politiques (la composition du parlement et des coalitions gouvernementales) et sociaux tels que l'influence et l'homogénéité des syndicats de fonctionnaires. C'est pourquoi ce point ne sera pas développé ici plus avant.

ii) Grands principes et règles

Ce cadre juridique minimum devrait au moins établir les principes et règles suivants :

a) la définition du CCS et de ses relations avec le reste de la fonction publique, en particulier en ce qui concerne les relations entre fonctionnaires spécialistes et généralistes (voir sous iii) ; 
b) la définition de conditions minimales d'admissibilité et l'établissement clair d'institutions et/ou du pouvoir de vérifier les qualifications des candidats. Cela implique aussi que soit précisé si seuls des fonctionnaires de carrières peuvent devenir membres du CCS, et dans le cas contraire, quel est le pourcentage maximal de nominations politiques ou temporaires dans le corps. Dans ce cas, la loi devrait également préciser si certains emplois ne peuvent être occupés que par des fonctionnaires de carrière, ainsi que les critères à appliquer et les institutions compétentes pour établir une liste de ces emplois ;

c) la définition des responsabilités pour la sélection et la gestion des carrières, précisant quels sont les pouvoirs des différentes institutions du gouvernement central. Différents choix peuvent être effectués, comme le montrent les deux exemples qui suivent :

\section{Exemple 1}

\section{Pouvoir de décision}

\begin{tabular}{llccc} 
Sélection & Nomination & Évaluation & Promotion & Mutation $\begin{array}{c}\text { Mesures } \\
\text { disciplinaires }\end{array}$ \\
\hline \hline
\end{tabular}

\section{Institutions}

Chef de l'État X

\begin{tabular}{|c|c|c|c|}
\hline Parlement & & & \\
\hline Chef du gouvernement & & $\mathbf{X}$ & \\
\hline \multicolumn{4}{|l|}{ Conseil des Ministres } \\
\hline Ministres & & & \\
\hline $\begin{array}{l}\text { Chefs des } \\
\text { Administrations }\end{array}$ & $\mathbf{X}$ & & $\mathbf{X}$ \\
\hline
\end{tabular}

Jurys spéciaux $\quad \mathbf{X}$

Dans ce premier exemple, les nominations dans le CCS sont formellement de la compétence du chef de l'Etat, afin de souligner le fait que les membres du Corps sont au service du gouvernement en tant qu'institution et non au service d'un ministère ou du seul gouvernement en place. Les mutations sont décidées par le Chef de gouvernement, afin d'éviter que les ministres ne soient obligés de garder des collaborateurs immédiats qui ne soient pas en accord avec la ligne politique du gouvernement en place, mais ils restent dans l'administration active. La sélection est confiée à des jurys spéciaux, afin de garantir l'égal accès au CCS et la qualification professionnelle de ses membres, alors que l'évaluation et les mesures disciplinaires, de nature technique, sont du ressort des fonctionnaires qui dirigent l'administration concernée. La promotion dépend des ministres puisqu'elle peut conduire aux plus hauts emplois de l'administration : si la promotion dépendait de la fonction publique elle-même, il pourrait facilement arriver qu'un fonctionnaire ne correspondant pas aux besoins d'un ministre devienne son secrétaire général. 


\section{$\underline{\text { Exemple 2 }}$}

Pouvoir de décision

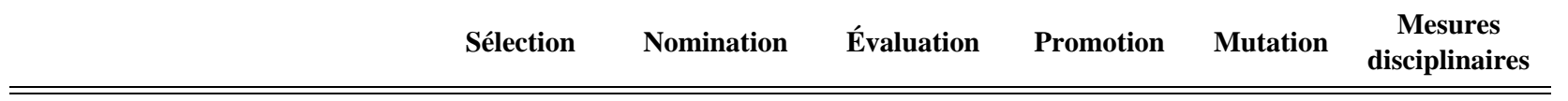

\section{Institutions}

Chef de l'État

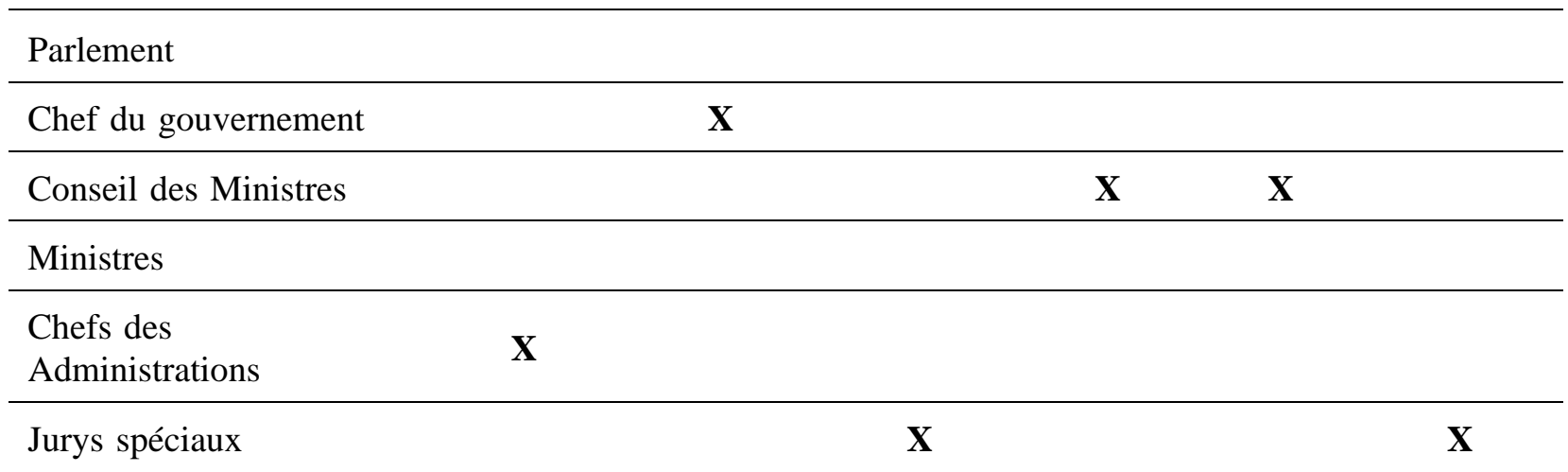

Dans ce second exemple, les nominations dans le CCS sont formellement de la compétence du chef de gouvernement, afin de souligner le fait que les membres du Corps sont au service du gouvernement en tant qu'institution et non au service d'un seul ministère. Les mutations et promotions sont décidées par le Chef de gouvernement, afin d'éviter que les ministres ne soient obligés de garder des collaborateurs immédiats qui ne soient pas en accord avec la ligne politique du gouvernement en place, mais ils restent dans l'administration active. Ceci devrait éviter la sectorialisation. La sélection est effectuée par les chefs des administrations, parmi les fonctionnaires possédant déjà les qualifications professionnelles nécessaires, alors que l'évaluation et les mesures disciplinaires sont du ressort de commissions spéciales afin de garantir la neutralité du système de carrière à l'égard de la politique partisane.

d) la mise en place d'institutions et/ou la fixation des compétences pour le développement de programmes de formation spécifiques, avec la définition d'un lien minimum entre la formation et l'admission dans le corps, ainsi qu'entre la formation et les avantages de carrière ;

e) l'établissement du principe d'une classification et description des emplois de l'administration, et la fixation des compétences pour établir des critères à cet effet et en vérifier l'application, de même que pour l'approbation de la liste des emplois inclus dans le système de CCS ;

f) la définition des principes du système de rémunération et de retraites du CCS et la mise en place d'institutions et/ou la fixation des compétences pour gérer ces systèmes.

\section{iii) Liens avec un statut général de la fonction publique}

Il ne paraît pas nécessaire qu'un pays ait déjà adopté une législation générale pour l'ensemble de la fonction publique avant de mettre en place un CCS. En fait, le nombre et la complexité des dispositions nécessaire pour le cadre juridique d'un CCS sont beaucoup moins importants que pour un statut général de la fonction publique. Cela signifie que l'établissement d'une réglementation pour un CCS serait plus rapide 
que pour l'ensemble de la fonction publique. La mise en place d'un CCS, limitée à quelques centaines d'emplois, peut d'ailleurs aider à expérimenter en matière de système de fonction publique avant de procéder à des choix définitifs.

Néanmoins, la mise en place d'un cadre juridique pour le CCS devrait n'être que provisoire, et les liens avec un futur système général devraient être envisagés aussi clairement -- même si ce n'est pas avec autant de détails -- qu'avec un système déjà existant. Les principaux problèmes à résoudre sont les suivants :

a) le cadre juridique du CCS et le statut général de la fonction publique doivent établir clairement les conditions et conséquences d'un passage du système général au CCS et vice-versa. Des principes de correspondance de rémunérations doivent être fixés afin que l'admission au CCS ne puisse pas conduire à une diminution de salaire net. Des principes minimaux de compensation doivent être fixés pour ceux qui retourneraient le cas échéant du CCS au système général ;

c) les deux cadres juridiques devraient préciser si les systèmes de rémunérations et de retraites du CCS font partie du système général de la fonction publique ou s'ils sont spécifiques au CCS. Dans ce dernier cas des règles doivent être développées pour permettre aux fonctionnaires de transférer leurs droits à pensions lorsqu'ils passent d'un système à l'autre ;

d) des mécanismes doivent être prévus pour assurer la coordination entre le cadre juridique du CCS et le statut général de la fonction publique, en particulier pour les cas de révisions partielles ;

e) des décisions doivent être prises en ce qui concerne les différentes carrières des généralistes et spécialistes.

\subsection{Un système de recrutement et de gestion de carrière}

Le système de recrutement et de gestion de carrière est probablement la composante essentielle d'un CCS. Il doit être construit avec soin, en prenant en compte toutes les specificités du pays et en laissant la place à une évaluation et à des corrections après quelques années d'expérience. Six éléments au moins doivent être pris en compte dans la construction d'un système de CCS :

\section{La taille du CCS}

La taille du CCS sera la résultante de la taille totale de la fonction publique de l'Etat et de l'étendue des emplois administratifs supérieurs inclus dans le CCS. La taille nette du CCS (nombre de membres ou d'emplois concernés) doit être suffisamment importante pour permettre la flexibilité dans l'allocation des emplois aux individus (quelques centaines), mais suffisamment réduite pour permettre le développement d'un esprit de corps (pas plus de quelque milliers). Dans tous les cas, il devrait y avoir une phase de transition avant que le CCS n'atteigne sa taille définitive ${ }^{4}$.

Ceci implique une clarification du système des emplois de la fonction publique ainsi qu'un certain nombre de choix :

\footnotetext{
${ }^{4}$ Afin de permettre des comparaisons : le SES fédéral américain comprend environ 8000 membres $(0.2$ pour cent de l'ensemble de la fonction publique fédérale); la dirigenza italienne représente 2 pour cent du total de la fonction publique ; en Suède, un programme de développement spécial a été expérimenté pour les 200 à 300 directeurs généraux d'agences, qui représentent moins d'1/1 000 de l'ensemble de la fonction publique.
} 
a) la clarification de ce que sont les emplois politiques ou discrétionnaires et de ce que sont les emplois permanents ; il est clair que les ministres occupent des emplois politiques, mais le statut des conseillers de ministres et des emplois supérieurs de ministères (tels que secrétaires généraux et directeurs) peut varier de pays à pays ;

b) décider combien de grades ou rangs doivent être inclus dans le $\mathrm{CCS}^{5}$. Le nombre de rangs peut varier d'un ministère à un autre ou d'un établissement public à un autre. Etant donné que tout ministère ou établissement serait conduit, pour des raisons de prestige, à inclure un maximum de rangs, il s'agit clairement ici d'un décision politique qui doit être prise par le Conseil des ministres ou le Premier ministre. Néanmoins, pour assurer le plus de transparence possible, il est à recommander d'avoir un système relativement homogène pour l'ensemble des ministères ;

c) décider si le CCS devrait s'étendre aux emplois de direction des entreprises publiques ou être réservé aux départements ministériels et établissements publics administratifs. Les possibilités d'évolution (privatisation d'entreprises publiques, transformations d'établissements publics en entreprises publiques) doivent être prises en considération ;

d) décider quels emplois doivent rester hors du CCS. Ce serait clairement le cas des emplois militaires dans le ministère de la Défense et d'autres ministères le cas échéant. Un liste claire d'emplois de spécialistes pourrait aussi être exclue du système. En tout état de cause, un choix clair devrait être fait, et rendu public, pour préciser si de telles exclusions reposent exclusivement sur des critères techniques ou également sur des critères politiques. Afin d'établir la confiance nécessaire à l'intérieur de la fonction publique, de telles décisions devraient pour le moins être de nature gouvernementale et non laissées aux ministres individuellement, mais elles n'ont pas besoin d'être établies par la loi.

ii)

\section{Critères d'admission}

Etant donné qu'un système de CCS est fondé sur la séparation entre le grade et l'emploi, il faut distinguer entre l'admission dans le corps et la nomination à un emploi. Cette dernière est liée par des règles constitutionnelles et implique en général une décision du Conseil des ministres ou d'un ministre, alors que l'admission dans le corps devrait être contrôlée par une institution spécialisée et autonome. Ceci signifie que la nomination sur un emploi du CCS ne peut concerner que des candidats déjà membres du CCS ou des personnes remplissant les conditions pour l'admission au CCS.

Ce devrait normalement être le rôle de l'institution de contrôle que d'établir des critères détaillés d'admission, prenant en compte les études, l'expérience et la réussite de programmes spécifiques de formation. Une ou des institutions particulières devraient être créées pour vérifier que les candidatures obéissent à ces critères.

Quoi qu'il en soit, des critères généraux d'admission doivent être précisés dans le cadre juridique du CCS, ce qui à nouveau implique des choix :

${ }^{5}$ Le $S E S$ fédéral américain, par exemple, couvre les trois rangs d'emplois les plus élevés du système administratif (si l'on exclut les emplois auxquels il ne peut être nommé qu'avec l'approbation du Sénat, qui ne peuvent être considérés comme des emplois politiques). En Allemagne, d'un autre côté, le service supérieur comprend tous les emplois d'encadrement et peut même inclure des emplois sans responsabilités d'encadrement, qui sont les points d'entrée dans la carrière. 
a) l'admission au CCS n'est-elle possible que pour des fonctionnaires de carrière déjà titulaires ou le Corps est-il ouvert à des personnes extérieures, venant du secteur privé ou nommées sur critères politiques ?

b) dans le premier cas, quelle est la durée nécessaire de service dans la fonction publique pour être admissible?

c) dans le deuxième cas, comment déterminer la correspondance entre l'expérience à l'intérieur et à l'extérieur de la fonction publique ? Une telle admission au CCS est-elle de nature permanente ou bien est-elle limitée à la durée de la nomination à un emploi particulier (et quelles sont les conséquences en matière de systèmes de retraite) ?

d) la formation liée à l'admission doit-elle avoir lieu avant qu'une nomination ne soit effective ou bien après une période de travail ? Y a-t-il seulement une institution chargée du développement et de la mise en ouvre des programmes de formation pour les candidats au CCS ?

e) est-il possible de réintégrer le CCS après l'avoir quitté volontairement ou involontairement ?

f) quel est le sort de nominations d'urgence sur des emplois du CCS ?

Différents choix peuvent être effectués et il faut souligner que, en particulier en période de transition, il est à recommander de prévoir différentes voies d'entrée dans le Corps, à condition que les règles relatives à chaque mécanisme d'entrée soient simples et transparentes.

\section{iii) Principes relatifs aux mutations}

Etant donné qu'un CCS doit permettre la flexibilité dans les nominations et, à défaut de les neutraliser totalement, au moins structurer les ingérences de la politique partisane, il est nécessaire de prévoir des principes pour les mutations. Là aussi il faut effectuer des choix :

a) les mutations d'emploi de CCS sont-elles entièrement à la discrétion du ministre ? même si c'est le cas, un système de recours permettrait d'éviter qu'une décision politique ne soit déguisée en sanction disciplinaire ou de gestion insuffisante ;

b) les mutations sont-elles soumises à des limites de temps (délais de préavis, moratoire temporaire sur les mutations lorsqu'un nouveau ministre prend son poste) ${ }^{6}$ ? ces délais devraient-ils être communs à l'ensemble du gouvernement ou doivent-ils prendre en compte la diversité des départements ministériels ? dans ce dernier cas, la décision ne devrait pas appartenir individuellement aux ministres, mais faire au moins l'objet d'une décision en Conseil des ministres ;

c) les mutations sont-elles soumises à d'autres conditions procédurales ou techniques ? comment et par qui ces conditions sont-elles fixées et contrôlées ?

\footnotetext{
${ }^{6}$ Dans le SES fédéral américain, un moratoire de 120 jours doit être respecté lorsqu'une nouvelle administration s'installe après l'élection présidentielle. Ceci est censé permettre une meilleure connaissance mutuelle avant les décisions concernant d'éventuelles mutations.
} 
d) qu'advient-il des cadres mutés s'ils ne sont pas immédiatement nommés sur un autre emploi vacant ? afin de répondre à cette question essentielle, il faut vérifier les emplois inclus dans le CCS afin de voir s'il y a un nombre suffisant d'emplois en dehors de ceux qui nécessitent une relation particulièrement étroite avec le ministre ; sinon, un système de "réservoir" doit être imaginé afin d'occuper utilement les membres du CCS pendant la période où ils ne trouvent pas d'emplois vacants leur convenant dans l'administration active ${ }^{7}$;

e) doit-on fixer une limite maximale d'occupation d'un emploi ? dans beaucoup de pays occidentaux l'on considère qu'au delà de cinq ans dans une même fonction, un manque de flexibilité ou d'imagination apparaît, ou même pire ;

f) quel est le type d'institution centrale la mieux à même de gérer ces mutations ?

Les questions qui viennent d'être posées montrent clairement que pour permettre des décisions sur ces éléments du CCS il faut procéder à une analyse démographique de la fonction publique existant dans le pays concerné.

\section{iv) Principes relatifs à la carrière}

L'un des éléments les plus délicats à concevoir lors de la mise en place d'un CCS est la structure appropriée du système de carrière, incluant les éléments susceptibles de motiver les membres du CCS pour qu'ils restent dans le Corps. La mise en place et le fonctionnement d'un CCS sont un bon moyen pour garantir un bon recrutement de jeunes fonctionnaires, s'il montre clairement qu'il y a des perspectives de carrière intéressantes pour des cadres administratifs intelligents et motivés. Pour ce faire il faut un lien clair entre la promotion et l'évaluation.

Si le CCS est limité aux emplois supérieurs de l'administration et si la taille totale de la fonction publique est peu élevée, il y a clairement un risque de décourager quelques uns des meilleurs membres du Corps après quelques années de service.

Ceci entraîne la nécessité de mettre en place une institution de gestion centrale chargée de coordonner les carrières selon des critères communs de promotion aux emplois les plus importants. La seule prise en compte des évaluations et de mécanismes de marché pourrait être pertinente pour des pays disposant d'un marché de l'emploi des cadres supérieurs flexible, ainsi que d'un faible différentiel de salaires entre les secteurs public et privé ${ }^{8}$. Ce serait extrêmement dangereux dans les PECO, où les cadres supérieurs sont une ressource rare en général et où la tendance à ce que les meilleurs éléments de la fonction publique fuient l'administration pour gagner le secteur privé n'a généralement qu'été trop forte.

\footnotetext{
${ }^{7}$ Un exemple intéressant est celui du Programme d'Affectations Provisoires du gouvernement canadien, qui permet aux titulaires des emplois supérieurs des ministères d'être placés en détachement temporaire, avec une mission de formation ou de recherche dans les universités ou les centres administratifs de formation ou de recherche.

${ }^{8}$ C'est manifestement la philosophie du Senior Executive Service de Nouvelle-Zélande.
} 
Une solution complémentaire pourrait être l'organisation de la mobilité entre les emplois de l'administration et ceux des entreprises publiques. L'on peut aussi mentionner dans ce contexte les réglementations de la fonction publique permettant un accès facile aux carrières politiques ${ }^{9}$.

\section{v) Des institutions pour l'admission, les mutations et la gestion des carrières}

Les points qui précèdent devraient avoir clairement démontré le besoin d'institutions spécifiques pour gérer le CCS. Il faut trouver un équilibre entre une inutile complexité des institutions et la prise en compte de besoins variables de décisions prises par des organismes indépendants ou au contraire par les chefs de départements ministériels ou établissements publics.

Les nominations à des emplois du CCS devraient être de la compétence des membres du gouvernement, mais les décisions relatives à l'admission au CCS de même que le contrôle des mutations devraient être du ressort d'un organisme autonome, ou du moins non-partisan ; la gestion d'ensemble du CCS devrait probablement relever des services du Premier ministre, ou d'un ministère de la fonction publique, si une telle institution existe.

Afin de faciliter la mise en place d'un CCS, il apparaît utile de créer une institution centrale qui permette la gestion formelle et informelle des carrières, en particulier parce qu'elle fournirait les occasions d'emploi des membres du Corps qui ne peuvent, à un certain moment, être en fonction dans les emplois supérieurs des ministères, parce qu'il leur manque la confiance politique du ministre. Il peut s'agir d'un centre de formation et de recherche, où les membres du corps pourraient travailler après un changement de gouvernement conduisant à leur mutation. Ce peut aussi être un organisme d'audit, de contrôle ou d'inspection, ou même un tribunal administratif, comme le Conseil d'Etat, la Cour des Comptes, ou l'Inspection générale des Finances en France. Bien que le rôle premier et officiel d'une telle institution soit sa fonction de contrôle, il s'agit d'un système très efficace de formation "sur le tas". Ses membres ont une vue d'ensemble de l'ensemble de l'appareil administratif du fait de leurs fonctions dans l'institution. De ce fait ils peuvent aisément occuper des emplois supérieurs de ministères, et ils ont également une base de repli disposant de suffisamment de prestige et de travail intéressant, pour le cas où un nouveau ministre veut changer les titulaires de ces postes.

\section{vi) La transparence pour pourvoir aux vacances}

Le mécanisme le plus important pour assurer une bonne gestion du CCS comme pour favoriser le développement d'un soutien à ce système dans la fonction publique consiste à assurer la transparence du processus en ce qui concerne l'accès au CCS. De plus un système de publicité des vacances d'emplois, permettant une concurrence des membres les mieux qualifiés du Corps pour la plupart des emplois vacants doit être mis en place. S'il est vrai que les ministres pourraient ressentir un tel système comme une contrainte, il devrait être possible de les convaincre qu'il n'a pas pour objectif de limiter leur possibilité de choisir les titulaires d'emplois supérieurs, mais bien plutôt de leur offrir un choix plus large de candidats appropriés et compétents parmi lesquels ils pourraient choisir les personnes avec lesquelles ils aimeraient travailler.

\footnotetext{
${ }^{9}$ En Allemagne et en France, par exemple, les fonctionnaires ont le droit de se présenter aux élections. S'ils sont élus, ils ne doivent pas démissionner de la fonction publique, mais sont simplement mis automatiquement en détachement provisoire. S'ils ne sont pas réélus ils peuvent donc retourner dans la fonction publique où ils ont gardé leurs droits à la retraite et à l'avancement. Ceci explique pourquoi un nombre important d'hommes politiques français connus sont membres des grands corps.
} 
Les discussions qui ont lieu aux États-Unis et dans quelques autres pays où existe un Senior Executive Service, ou bien où l'on envisage d'en installer un, montre qu'une question très importante ne reçoit souvent pas de réponse claire : celle des responsabilités.

Du fait de l'existence de certains principes contradictoires parmi les objectifs et dans la structure de ces Corps, des questions se posent quant à la responsabilité des membres du corps : sont-ils responsables devant leur supérieurs immédiats ou devant le gouvernement, ou devant le Parlement ou bien devant leurs "clients", ou sont-ils responsables uniquement devant la loi et la justice ?

Dans la plupart des cas, ce débat est la conséquence de l'influence de certaines opinions managérialistes simplistes, qui ont dominé le débat public sur la fonction publique dans les années quatre-vingt. Etant donné l'influence que ces opinions ont aussi eu dans certains PECO, des prises de position claires quant aux responsabilités des membres du CCS devraient être adoptées par les gouvernements concernés, et trouver leur place dans le cadre juridique du système de même que dans les programmes de formation.

\subsection{Une formation spécifique}

L'une des caractéristiques particulières des CCS est l'importance qu'ils doivent donner au développement des cadres et des candidats. L'usage de ce vocabulaire au lieu des concepts plus classiques de formation et études se réfère à des options philosophiques et aux contraintes techniques inhérentes aux CCS.

\section{i) La prise en compte des contraintes techniques}

La formation des membres et futurs membres du CCS est soumise aux mêmes contraintes que la formation de l'ensemble de la fonction publique dans les PECO (rareté du matériel pédagogique spécialisé, développement insuffisant des programmes et méthodes de formation dans le secteur public, etc.).

Mais il y a aussi des contraintes plus spécifiques, qui découlent de la nature du CCS, et que l'on rencontre également dans les pays les plus développés : les clients de tels programmes ne disposent que d'un temps très limité pour la formation, du fait qu'ils sont en général les hauts fonctionnaires ou cadres privés les plus efficaces. C'est pourquoi les programmes de développement doivent s'appuyer en très grande partie sur des méthodes d'apprentissage par l'action, dans lesquelles la partie la plus longue du programme est mise en œuvre pendant l'exercice des fonctions, avec des sessions intermédiaires de préparation et de suivi, et en relation étroite avec des conseillers ou tuteurs. L'un des avantages de cette méthode est que la plupart des personnes formées deviennent elles-mêmes des formateurs - pour leur propre personnel pendant le déroulement du programme de développement, et pour les nouveaux venus dans le Corps lors des années suivantes.

\section{ii) L'adaptation des programmes de développement aux domaines qui nécessitent le plus d'impulsion professionnelle}

Le contenu final des programmes de développement des cadres supérieurs de l'administration doivent manifestement être imaginés pays par pays, en prenant en compte leur diversité. 
Néanmoins un travail commun aux institutions de formation des PECO pourrait permettre d'établir un cadre commun utile, indiquant le type de thèmes à traiter et de méthodes à utiliser ${ }^{10}$.

\section{iii) Le lien entre l'évaluation et le développement des carrières}

Les programmes de formation des membres du CCS devraient être fortement individualisés. Ils peuvent être développés au mieux après des entretiens d'évaluation, dans un processus impliquant le cadre supérieur ou candidat à l'entrée dans le Corps, l'unité responsable de la gestion de la formation, et le ou les supérieurs hiérarchiques directs. Ils sont structurés de façon à améliorer le cadre supérieur dans les domaines où il (elle) a le sentiment que son efficacité est insuffisamment développée. D'un point de vue idéal, le programme de développement devrait conduire à une nouvelle évaluation et à un développement de carrière dès qu'un nouvel emploi approprié est vacant.

\section{iv) Des stratégies spécifiques pour les PECO}

La mise en place de CCS dans les PECO nécessitera sûrement des stratégies spécifiques. Il faudra prendre en compte le besoin d'attirer de nouvelles générations dans la fonction publique, ce qui conduira probablement à la mise en place d'un système différencié d'études pour les candidats externes et de formation par l'action pour les membres du Corps et candidats internes.

La rareté des ressources conduira aussi probablement à tirer parti de la coopération internationale pour la conception et le suivi des programmes de développement. En tout état de cause, leur mise en œuvre devrait être autant que possible confiée à des institutions et formateurs locaux. Afin de faciliter l'entrée de futurs fonctionnaires dans le CCS, le rôle des programmes d'administration publique des universités ou instituts spécialisés devrait être examiné et de nouveaux programmes développés.

\subsection{L'analyse et la description des emplois}

Afin de permettre la mobilité entre ministères et établissements publics, de même que pour délimiter l'ampleur du CCS, les départements et établissements devraient se livrer à une analyse des emplois supérieurs. Ceci pourrait être fait par les institutions qui géreront le CCS de manière centrale, en coopération avec les ministères et les titulaires de ces emplois, afin de garantir l'utilisation d'une méthodologie commune à l'ensemble de l'administration.

Cet effort devrait se concrétiser par deux produits principaux :

-- une liste des emplois à inclure dans le CCS, et

-- des descriptions d'emplois (ou profils de postes) qui pourront être utilisées pour la fixation d'objectifs pour l'évaluation, pour analyser les besoins de formation du point de vue de l'organisation ainsi que pour la publicité des vacances d'emplois. Les descriptions d'emplois devraient évoluer avec le service et être périodiquement révisées. Cela signifie que l'analyse des emplois n'est pas une opération limitée, liée à la seule mise en place d'un CCS, mais qu'elle devrait devenir une partie du fonctionnement courant du système.

${ }^{10}$ La première École d'été du Réseau des Instituts et Écoles d'administration publique des PECO (NISPACEE), qui a lieu à Portoroz (Slovénie) en août 1994, a permis l'établissement d'un projet de ce type (voir l'Annexe II). 
Les promoteurs de "Senior Executive Systems" aux États-Unis et ailleurs ont essayé d'y inclure de manière plus ou moins extensive un système de rémunération au mérite. Ceci a été présenté comme un facteur essentiel de motivation et de dynamisme pour les membres du corps. Néanmoins l'expérience montre, en particulier dans le cas du SES fédéral américain, que la rémunération au mérite est devenue l'un des éléments essentiel de mécontentement pour les membres du Corps. Il y deux raisons principales à cela. D'une part, quelques-unes des promesses qui étaient associées aux réformes mettant en place le SES n'ont pas été tenues du fait du changement de gouvernement et du manque de moyens financiers. D'autre part, et ceci est sans doute plus important, l'on s'est mis à soupçonner que les primes étaient distribuées non pas en fonction de la qualité du service, mais en fonction de préférences personnelles ou politiques, car ces primes étaient réparties par les supérieurs politiques des fonctionnaires, dans une période où ceux-ci étaient les témoins d'un nouvel accroissement des nominations politisées. La leçon à tirer de ces expériences est l'importance d'une gestion continue d'un CCS. Il n'est pas à recommander de se contenter de mettre le corps en place.

L'expérience de la plupart des pays qui ont essayé d'introduire un système de rémunération au mérite montre qu'il faut constamment choisir entre d'un côté des critères de rémunération automatique, qui conduisent à la démotivation de nombreuses personnes du fait que ces critères ne tiennent pas suffisamment compte de la qualité réelle du service, et de l'autre la répartition discrétionnaire par les chefs de service, qui conduit au développement de soupçons quant à l'objectivité de ce chef, en particulier s'il a été nommé sur critères politiques. Il faudra en tenir compte dans la mise en place des système de paiement et la formulation des conditions de rémunération.

Un autre élément doit être pris en compte : si l'un des principaux objectifs de la mise en place d'un CCS est la promotion d'une élite administrative dotée d'un esprit de corps, la concurrence entre les membres de ce corps doit rester relativement limitée. Il est plus important de souligner les valeurs et comportements communs que les différences.

Ceci conduit au développement d'un système de rémunération comprenant trois éléments qui doivent coexister :

i) Une partie importante de la rémunération doit être liée au grade à l'intérieur du corps. En cas d'absence d'emplois vacants ceci permet néanmoins des promotions (à l'intérieur du corps) à conséquences financières importantes, même si la structure des emplois est très rigide. Cette partie de la rémunération ne doit pas être automatiquement liée à l'ancienneté : une partie de l'avancement peut être liée à la réussite de périodes de formation et à des évaluations positives, et peut être gérée de manière centralisée avec la participation de représentants élus des membres du corps.

ii) Une autre partie importante de la rémunération doit être liée à l'emploi effectivement occupé par chacun des membres du corps. Des descriptions d'emplois fiables sont indispensables pour que ceci fonctionne bien, sans qui les critères mis en œuvre risquent d'être des critères de statut social, liées à l'importance politique des ministres ou chefs d'établissements.

iii) Une troisième partie de la rémunération peur consister en récompenses particulières, liées ou non à la qualité du service. C'est sans doute la partie du système la plus difficile à mettre en place, du fait qu'elle doit être liée à l'évaluation de la manière de servir, effectuée par le chef (politique) du service. La seule solution permettant de limiter les soupçons semble être un système standardisé et centralisé d'évaluation, assorti de péréquations entre les ministères, et conduisant à la formulation de recommandations aux ministres. L'expérience montre qu'il y a alors un risque que le système de primes ne prenne un caractère automatique et ne perde ses liens avec la qualité réelle du service. Plusieurs études sociologiques dans les 
pays occidentaux ont montré que les récompenses d'ordre financier sont importante pour attirer de jeunes diplômés de l'enseignement supérieur dans la fonction publique. Par contre elles ne jouent qu'un rôle mineur -- ou pas du tout -- dans la motivation des hauts fonctionnaires : un système clair et sûr de retraite est souvent considéré comme beaucoup plus important à ce niveau que des primes particulières.

Il faut accorder une attention particulière à la structure et à la transparence des récompenses de type monétaire et non monétaire : il paraît fondamental dans les PECO d'éviter de donner au grand public et au reste de la fonction publique l'impression qu'un CCS n'est qu'une nouvelle version de la nomenklatura.

\section{Conclusions}

Un Corps de cadres supérieurs (CCS), c'est-à-dire un système structuré et reconnu de gestion du personnel pour les emplois supérieures à caractère non politique (les emplois de cadres publics supérieurs) dans l'administration, peut être une réforme essentielle afin de permettre le développement des fonctions de gestion transversales à l'administration, pour augmenter la qualité et l'efficacité du travail du gouvernement central et de son mode de prise de décision, et pour améliorer le processus général de gestion des ressources humaines dans l'administration. Il peut permettre d'augmenter la compétence professionnelle de la haute fonction publique en évitant des ingérences de la politique partisane dans les procédures de sélection et la gestion des carrières et ainsi augmenter les possibilités de choix des ministres qui veulent disposer de collaborateurs efficaces mais également sensibles aux aspects politiques des dossiers.

Un tel système est fondé sur une fonction publique de carrière, fournissant des cadres formés et expérimentés pour pouvoir être nommés aux emplois de cadres publics supérieurs. Le corps est géré de manière centrale par des institutions et procédures appropriées afin de développer la stabilité et le professionnalisme du groupe central de cadres supérieurs, mais aussi pour permettre la flexibilité nécessaire pour répondre aux changements dans la composition du gouvernement. Bien que le présent document pose un grand nombre de questions auxquelles il faut répondre pour pouvoir mettre en place un CCS, un tel système n'a pas besoin d'être très compliqué ; il peut être fondé sur un modèle robuste et simple adapté aux besoins immédiats d'un pays et pouvant être développé par la suite afin de prendre en compte les développements de l'administration publique et la réforme de la fonction publique. 


\section{ANNEXE I}

\section{BIBLIOGRAPHIE SÉLECTIVE}

BAN, Carolyn and INGRAHAM, Patricia eds. (1984), Legislating Bureaucratic Change: The Civil Service Reform Act of 1978, Albany State University Press, Albany, New York.

BUCHANAN, Bruce (1981), "The Senior Executive Service: How we Can Tell if it Works?", Public Administration Review, No. 41, pp. 349-358.

CULLEN, R.B. (1986), "The Victorian Senior Executive Service: A Performance Based Approach to the Management of Senior Managers", Australian Journal of Public Administration, Vol. XLV, No. 1 (March).

HECLO, Hugh (1983), "A Government of Enemies", The Bureaucrat, No. 12, pp. 12-14.

INSTITUT INTERNATIONAL DES SCIENCES ADMINISTRATIVES (1988), La haute fonction publique en Belgique et dans les pays industrialisés -- Recrutement, carrière et formation, IISA, Bruxelles.

KESSLER, M-C. (1994), Les grands corps de l'État, PUF, Paris.

NEWLAND, Chester A. (1988), "The American Senior Executive Service: Old Ideas and New Realities", International Review of Administrative Sciences, No. 4, p. 699.

SULEIMAN, Ezra N. (1984), Bureaucrats \& Policy-Making, Holmes \& Meyer, New-York.

ZILLER, Jacques (1993), "Les systèmes politico-administratifs de l'Europe des Douze", Administrations comparées, Montchrestien, Paris.

ZILLER, Jacques (1988), "L'accès à la fonction publique dans les États de la Communauté européenne", Égalité et mérite, Bruylant, Bruxelles. 


\section{RECOMMANDATIONS DU RÉSEAU NISPACEE POUR UN PROJET DE PROGRAMME DE FORMATION POUR LES CADRES PUBLICS SUPÉRIEURS}

À l'occasion de la première École d'été consacrée au développement de programmes par le Réseau des instituts et écoles d'administration publique d'Europe centrale et orientale (NISPACEE), les participants ont discuté de manière approfondie la formation permanente.

Le groupe de travail a produit une liste d'environ 25 thèmes à prendre en considération et a dégagé un consensus sur les domaines d'enseignements suivants :

1. DROIT

- Utilisation des banques de données

- Familiarisation avec les sources du droit

- Techniques d'interprétation pour l'analyse de textes

- Cadre juridique

- Protection des droits de l'Homme

- Déréglementation

- Système judiciaire

- $\quad$ Structure et procédures du droit administratif et constitutionnel

2. BUDGET ET FINANCES

- $\quad$ À quoi doit ressembler une bonne procédure budgétaire

- Techniques budgétaires et financières

- Comment appréhender et comprendre la procédure budgétaire

- Rôle des institutions telles que Trésor, Ministère des finances, institutions externes

- Détermination et contrôle des coûts

- Marchés publics (procédures et droit)

3. COMMUNICATION

- Communication interne à l'administration:

- conduite de réunions

- gestion et circulation de l'information

- stratégies de communication interne

Communication externe à l'administration:

- aptitudes à l'écriture et à la présentation

- la transparence et ses éléments dans le public (incluant les minorités)

- relations publiques

- relations avec les media 


\section{RôLE ET RESPONSABILITÉS DES CADRES}

- Pour la planification

- Pour la fixation des priorités

- Pour la fixation des buts

- Pour la détermination de l'équilibre entre politiques, programmes et ressources

- Pour donner des avis

- Pour que les choses se passent

\section{GESTION DES RESSOURCES HUMAINES}

Aspects descriptifs -- statut de la fonction publique, catégories d'emplois

- Politiques de recrutement, d'avancement, de qualification

- Systèmes et conditions de rémunérations

- Évaluation du rendement

- Dossiers du personnel

- Motivation et récompense

6. GESTION DES SERVICES ET DES OPÉRATIONS, DES BUREAUX

Aptitude à l'analyse et à l'amélioration du service rendu

Amélioration du service public:

. opérations

- comportement

- Guichets uniques

- Formulaires conviviaux

- Amélioration de la gestion des bureaux

- Éthique et corruption

7. SOLUTION DES CONFLITS, NÉGOCIATION

- Aptitudes inter-personnelles

- Coopération/concurrence

- Constitution d'équipes

- Culture administrative

- Relations entre comportement et stress

- Comparaison des comportements (dans d'autres cultures et secteurs publics)

Comportements dans le secteur public et nouveaux comportements du secteur privé. 